\title{
The Effect of Particle Drag and Wall Absorption on Mass Transfer in Concentric Annulus Flows
}

\author{
B. Umadevi, ${ }^{*}$ P.A. Dinesh, ${ }^{\dagger}$ Indira R. Rao ${ }^{\ddagger}$ and C.V. Vinay*
}

\begin{abstract}
The effects of the irreversible boundary reaction and the particle drag on mass transfer are studied analytically in concentric annulus flows. The solution of mathematical model, based on the generalized dispersion model brings out the mass transport following by the insertion of catheter on an artery in terms of the three effective transport coefficients, viz., the exchange, convection and diffusion coefficient. A general expression is derived which shows clearly the time dependent nature of the coefficients in the dispersive model. The complete time dependent expression for the exchange coefficient is obtained explicitly and independent of velocity distribution in the flow; however it does depend on the initial solute distribution. Because of the complexity of the problem only asymptotic large time evaluations are made for the convective and diffusion coefficients, but these are sufficient to give the physical insight into the nature of the problem of the effects of drag and absorption parameters. It is found that as absorption parameter increases exchange and convection coefficients will be enhanced, but diffusion coefficient will be reduced. After certain period of time exchange coefficient will be constant for different
\end{abstract}

\footnotetext{
* Department of Mathematics, JSS Academy of Technical Education, Bangalore -560060

t Department of Mathematics, MS Ramaiah Institute of Technology, Bangalore-560054; dineshdpa@mstit.edu (Corresponding Author)

‡Department of Mathematics, Nitte Meenakshi Institute of Technology, Bangalore-560064
} 
values annular gap. As the drag parameter increases convection and diffusion coefficients will be reduced. With the enhancement of catheter radius i.e., the annular gap will be reduced then the convection and diffusion coefficients will be decreased.

Key Words: Drag parameter, Wall absorption, Exchange coefficient, Convection coefficient, Diffusion coefficient.

\section{Introduction}

The dispersion of solute in physiological fluid flows is of practical interest. Many physiological situations involve inter phase mass transfer. The involvement of mass transfer in atherosclerosis has been investigated from two vantage points. One, it is a fact that the lipid component of the atherosclerotic plaque are largely derived from the plasma. Obviously, these lipids are not very soluble in an aqueous medium such as plasma, and they are transported in blood and lymph in association with protein carrier molecules called lipoproteins. Thus, there is a well founded interest in factors which control the influx of the lipoproteins and other macromolecules into the arterial wall.

The problem of natural convection mass/heat transfer in an annulus bounded by two horizontal cylinders has been a subject of great interest due to its wide technological applications like nuclear reactors, geothermal energy recovery, exothermic chemical reaction etc. (Tsui and Tremblay [9]), continuous annular chromatographic method used in separation of metals, sugars, proteins etc. (Carta et al. [3], Bloomingburg [2]). An example from clinical medicine is the dye or indicator dilution technique which is a common practice among the physiologists. In this technique, catheters are used to inject the dye and to withdraw blood samples for the purpose of measurements. Also the study of blood- tissue exchange in the physiological system is done by multiple indicator dilution technique. The dispersion of mass or heat in blood vessels is influenced by the conductive blood vessel walls as well as inserted catheter. Modeling of this situation is usually done by the considering the flow as the annular flow between the inserted 
catheter and blood vessel wall ( Sarkar and Jayaraman [7] , [8] Dash et al.[4], Back et al. [1] ). Sankarasubramanian and Gill [6] analyzed the dispersion of solute undergoing first order wall reaction in Poiseuille flow through a circular tube. Their generalized dispersion model gave rise to three effective coefficients, namely the convection, the diffusion and the exchange coefficients. Their approach was further developed by De Gance and Johns [5] to the case of a cylinder with arbitrary cross-section. The transport coefficients were found to depend upon a transverse average of the solute concentration. In the present study effect of solute drag on the convective diffusive mass transfer with wall absorption is analysed using a similar model to Sankarasubramanian and Gill [6].

\section{Mathematical formulation}

Figure 1 shows a schematic diagram of the concentric annular geometry with an outer tube as an artery of radius $R_{0}$ and an inner tube as a catheter of radius $\mathrm{k} R_{0}(\mathrm{k}<1)$. The flow in the concentric annulus is considered to be axi-symmetric, and the fluid is nonNewtonian as a dusty-viscous fluid

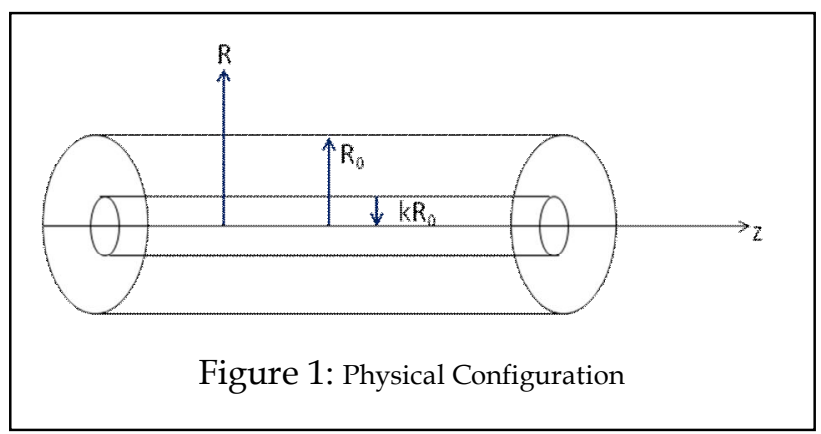

The governing momentum equations cylindrical coordinate system $(R, \theta, z)$ are given by

$$
\rho \frac{\partial W}{\partial t}=-\frac{\partial p}{\partial z}+\mu\left(\frac{\partial^{2} W}{\partial R^{2}}+\frac{1}{R} \frac{\partial W}{\partial R}\right)+K N(V-W)
$$




$$
N m \frac{\partial V}{\partial t}=K N(W-V)
$$

The no- slip boundary conditions are given by

$$
W=0 \quad \text { at } R=k R_{0} \text { and } R=R_{0}
$$

Where $\mu$ is the viscosity of the fluid, $\rho$ is the density of the fluid, $W$ is the velocity of the fluid phase, $V$ is the velocity of the dusty particle , $N$ is the number density of the particles, $K=3 \mu \pi d$ is the stokes drag term, $d$ is diameter of the dusty particle, $m$ is the mass of the each dusty suspended particle. $p$ is the pressure.

Assuming $\quad r=\frac{R}{R_{0}}, W=w(r) e^{-n t}, V=v(r) e^{-n t}, \frac{\partial p}{\partial z}=-P e^{-n t}$ in equations (1) and (2) we get the equations in non-dimensional form for the fluid phase and dusty phase

$$
\begin{aligned}
& \frac{d^{2} w}{d r^{2}}+\frac{1}{r} \frac{d w}{d r}+\omega^{2} w=P \\
& v(r)=\frac{1}{1-n \Gamma} w(r)
\end{aligned}
$$

where $\omega^{2}=v\left(n+\frac{K N}{\rho} \frac{n \Gamma}{1-n \Gamma}\right), \Gamma=\frac{m}{K}$.

Here $\omega$ is the drag parameter, $v$ is the kinematical viscosity, $\Gamma$ is the relaxation time, $P$ is the pressure gradient. $w$ is the nondimensional velocity of the fluid phase, $v$ is the velocity of the dusty particle phase.

Non-dimensional no-slip conditions are given by

$$
w=0 \text { when } r=k \text { and } r=1 .
$$


Mapana J Sci, 10, 1(2011) The Effect of Particle Drag and Wall Absorption

Solving (4) by using the boundary conditions given in (6)

The velocity profile for the fluid phase will be given by

$$
w(r)=\frac{P}{\omega^{2}}\left(A J_{0}(i \omega r)+B Y_{0}(i \omega r)+1\right)
$$

$A$ and $B$ are given in appendix.

The non-dimensional convective - diffusion equation in the cylindrical coordinate system $(r, \theta, z)$ for the dispersion in an annular flow of a solute with concentration is given by

$$
\frac{\partial c}{\partial t}+w(\tau, r) \frac{\partial c}{\partial z}=\frac{\partial^{2} c}{\partial r^{2}}+\frac{1}{r} \frac{\partial c}{\partial r}+\frac{1}{P e^{2}} \frac{\partial^{2} c}{\partial z^{2}}
$$

where the non-dimensional variables are

$$
c=C_{0} C, \quad z=\frac{D_{m}}{R_{0}^{2} w_{m}} Z, r=\frac{R}{R_{0}}, w=w_{m} W, \tau=\frac{D_{m} t}{R_{0}^{2}}
$$

$\tau$ is the non-dimensional time, $C_{0}$ is the reference concentration, $D_{m}$ is the molecular diffusivity, $w_{m}$ is the mean axial velocity, $R_{0}$ is the radius of the tube, and $P e=\frac{R_{0} w_{m}}{D_{m}}$ is the Peclet number. $w(\tau, r)$ represents the non-dimensional velocity in the axial direction $z$.

\section{Initial and boundary conditions}

The initial distribution of the solute assumed in a variable separable form is given by

$$
c(0, r, \theta, z)=B_{2}(z) B_{1}(r)
$$


with, $B_{2}(z)=\frac{\delta(z)}{a^{2} P e}$, where $\delta(z)$ denotes the Dirac delta function and $B_{1}(r)$ is defined as

$$
B_{1}(r)=1, k<r \leq \mathrm{a}, B_{1}(r)=0, \quad \mathrm{a}<\mathrm{r} \leq 1 .
$$

A number of boundary conditions at the flow boundary of the tubular reactors have been cited in the literature and are described in [3]. In the present model, we consider the reaction mechanism occurring at the wall $(r=1)$ of the tube so that

$$
\frac{\partial c}{\partial r}=-\beta c
$$

where $\beta$ is the non-dimensional wall reaction parameter. In addition, we have the boundary condition at

$$
\frac{\partial c}{\partial r}=0 \quad \text { at } \quad r=k
$$

describing the impermeable wall of inner tube.

\section{Mathematical method}

In order to solve the convection-diffusion equation (8) along with the associated sets of initial conditions (10), (11) and boundary conditions (12), (13), we express the concentration as

$$
c(\tau, r, z)=\sum_{n=0}^{\infty} f_{n}(\tau, r) \frac{\partial^{n} c_{m}}{\partial z^{n}}
$$

Where the average concentration $c_{m}$ is expressed as

$$
c_{m}=\frac{\int_{0}^{2 \pi} \int_{k}^{1} r c d r d \theta}{\int_{0}^{2 \pi} \int_{k}^{1} r d r d \theta}=\frac{2}{\left(1-k^{2}\right)} \int_{k}^{1} r c d r
$$


Mapana J Sci, 10, 1(2011) The Effect of Particle Drag and Wall Absorption

and the $f_{n}$ 's have to be determined using Eqs. (8) - (14). We assume that the distribution of mean concentration, $c_{m}$ can be described by the generalized dispersion model truncated after three terms as

$$
\frac{\partial c_{m}}{\partial t}=M_{0}(\tau) c_{m}+M_{1}(\tau) \frac{\partial c_{m}}{\partial z}+M_{2}(\tau) \frac{\partial^{2} c_{m}}{\partial z^{2}}
$$

The term $M_{0}(\tau)$ corresponds to the absorption parameter. This term arises because of the nonzero solute flux at the flow boundary $M_{0}(\tau)$ will be zero if there were no absorption in the wall. $M_{1}(\tau)$ and $M_{2}(\tau)$ correspond to the convective and dispersion coefficients, respectively. The convection coefficient $M_{1}(\tau)$ accounts for the velocity of the reactive tracer, and the dispersion coefficient $M_{2}(\tau)$ provides the modifications in the convective dispersion occurring because of absorption.

Substituting (14) in (8) and using (16) to evaluate $\partial c_{m} / \partial \tau$ and expressing $\partial^{n+1} c_{m} /\left(\partial \tau \partial z^{n}\right)$ in terms of $\partial^{n} c_{m} / \partial z^{n}$ and equating the coefficients of $\partial^{n} c_{m} / \partial z^{n}, n=0,1,2 \ldots$, we get the set differential equations for $f_{n}$ as

$$
\frac{\partial f_{n}}{\partial \tau}-\frac{\partial^{2} f_{n}}{\partial r^{2}}-\frac{1}{r} \frac{\partial f_{n}}{\partial r}+u(\tau, r) f_{n-1}-\frac{1}{P e^{2}} f_{n-2}+\sum_{n=0}^{\infty} f_{n-i} M_{i}=0, n=0,1,2 . .
$$

Where $f_{-1}=0 f_{-2}$ and $M_{n}(\tau)^{\prime}$ 's are described as

$$
M_{n}(\tau)=\frac{2}{\left(1-k^{2}\right)} \frac{\partial}{\partial r} f_{n}(\tau, 1)+\frac{\delta_{n, 2}}{P e^{2}}-\frac{2}{\left(1-k^{2}\right)} \int_{k}^{1} r u(\tau, r) f_{n-1} d r, n=0,1,2 \ldots . .(18)
$$

$\delta_{n, 2}$ denotes the Kronecker delta. The first term in (18) arises due to the first-order heterogeneous reaction at the outer wall of the tube. The coefficient $M_{o}(\tau)$ is first decoupled from the equation obtained from (17), 


$$
\frac{\partial f_{0}}{\partial \tau}=\frac{\partial^{2} f_{0}}{\partial r^{2}}+\frac{1}{r} \frac{\partial f_{0}}{\partial r}-M_{0} f_{0}
$$

From generalized dispersion model of Sankarasubramanian and Gill [6] the exchange coefficient is given by

$$
M_{0}(\tau)=-\frac{\sum_{n=0}^{\infty} \frac{A_{n}}{J_{1}\left(\mu_{n} k\right)} \mu_{n}\left(Y_{1}\left(\mu_{n}\right) J_{1}\left(\mu_{n} k\right)-Y_{1}\left(\mu_{n} k\right) J_{1}\left(\mu_{n}\right)\right) e^{-\mu_{n}^{2} \tau}}{\sum_{n=0}^{\infty} \frac{A_{n}}{J_{1}\left(\mu_{n} k\right)}\left(Y_{1}\left(\mu_{n}\right) J_{1}\left(\mu_{n} k\right)-Y_{1}\left(\mu_{n} k\right) J_{1}\left(\mu_{n}\right)\right) e^{-\mu^{2} \tau}}
$$

As $\tau \rightarrow \infty$ asymptotic representations for $M_{0}$

$$
M_{0}(\infty)=-\mu_{n}^{2}
$$

By solving Eq(18), the convection and dispersion coefficients under steady flow conditions are given by

$$
\begin{gathered}
M_{1}=-\frac{2}{\left(1-k^{2}\right)}\left(\beta f_{1}(1)+\int_{k}^{1} r w(r) f_{0} d r\right) \cdot=-\frac{\int_{\mathrm{k}}^{1} \mathrm{rw}(\mathrm{r}) \mathrm{E}_{0}\left(\mu_{0} \mathrm{r}\right) \mathrm{f}_{0}(\mathrm{r}) \mathrm{dr}}{\int_{\mathrm{k}}^{1} \mathrm{rE}_{0}\left(\mu_{0} \mathrm{r}\right) \mathrm{f}_{0}(\mathrm{r}) \mathrm{dr}}(22) \\
M_{2}-\frac{1}{P e^{2}}=\frac{-\int_{k}^{1} r\left(w(r)+M_{1}\right) f_{1}(r) E_{0}\left(\mu_{0} r\right) d r}{\int_{k}^{1} r E_{0}\left(\mu_{0} r\right) f_{0}(r) d r}
\end{gathered}
$$

\section{Results and Discussions}

In the present analysis, the development of the dispersive transport following the insertion of a catheter in to artery in a solvent flowing through concentric annulus with reactive outer wall has been studied, considering two phase of blood. The analysis uses the derivative expansion method proposed by 
Mapana J Sci, 10, 1(2011) The Effect of Particle Drag and Wall Absorption

Sankarasubramanian and Gill [6]. For each value of $\beta$ and $k$ the associated eigen values $\mu_{n}, n=0,1,2 \ldots$ are obtained by a standard root finding procedure. The objective is to evaluate the exchange coefficient $M_{0}(\tau)$, the asymptotic coefficient $M_{1}$ and the asymptotic dispersion coefficient $M_{2}$, and to find their dependence on the size of the annular gap ' $k$ ', the wall absorption parameter ' $\beta$ ' and the drag parameter ' $\omega$ '.

Exchange coefficient $M_{0}(\tau)$

The exchange coefficient $M_{0}(\tau)$ arises only due to the interphase mass transfer and hence it is not affected by the flow and is independent of drag parameter ' $\omega$ '.

The coefficient $M_{0}(\tau)$, accounting for this nonzero solute flux at the tube wall, will be negative in this problem to accont for the depletion of solute in the system. Figures 2,3 shows the variation of $M_{0}(\tau)$ with $\tau$ for different values of $\beta$. The figures show that the exchange coefficient reaches the asymptotic value of $-M_{0}(\tau)$ very fast when $\mathrm{a}=0.95$. But when $\mathrm{a}=0.5$ then $-M_{0}(\tau)$ is zero initially.

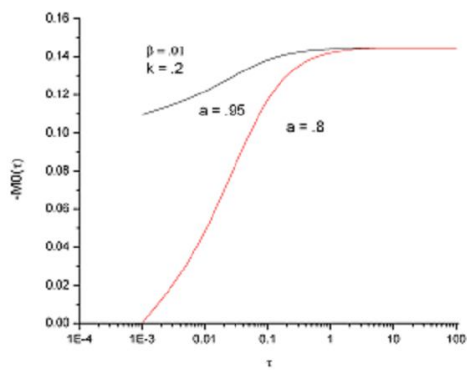

Figure 2

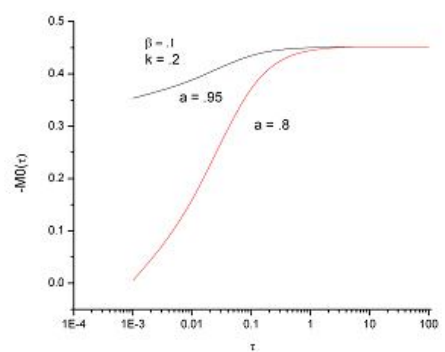

Figure 3 


\section{Variation of $-M_{0}(\tau)$ with time $\tau$}

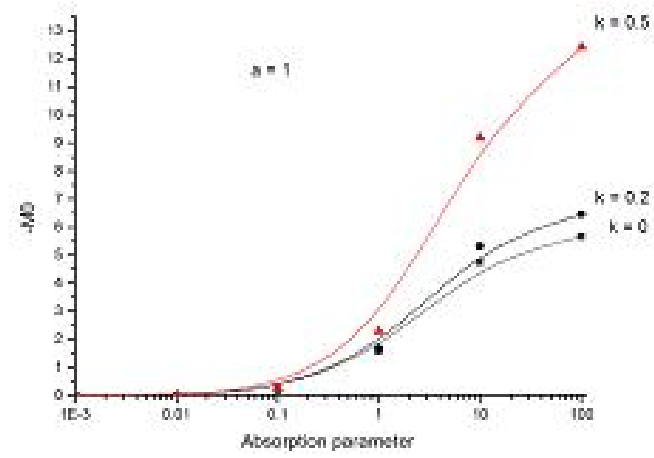

Figure 4: Variation of $-M_{0}$ with $\beta$ for different values of $k$

Figure 4 describes the variation of $-M_{0}$ (the exchange coefficient), there is more absorption of solutes at the tube wall in an annulus compared to the tubular flow. It is unaffected by drag parameter $\omega$ and increases with the increase in the value of the wall absorption parameter $\beta$.

\section{Asymptotic convection coefficient $M_{1}$}

From figures 5 and 6 , it can be seen that the asymptotic convective coefficient $-M_{1}$ decreases rapidly as the drag parameter $\omega$ increases successively by varying absorption parameter $\beta$ from 0.001 to 100 . That is the effect of particle drag on average velocity and also on asymptotic convective coefficient $-M_{1}$ is very much significant than the effects of annular gap $(k)$ and absorption parameter $\beta$.

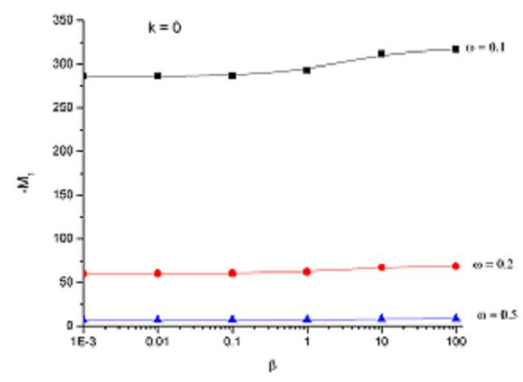

Figure 5

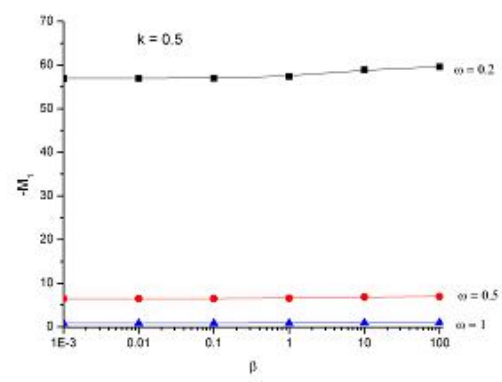

Figure 6 Variation of $-M_{1}$ 
Mapana J Sci, 10, 1(2011) The Effect of Particle Drag and Wall Absorption

\section{Asymptotic dispersion coefficient $M_{2}$}

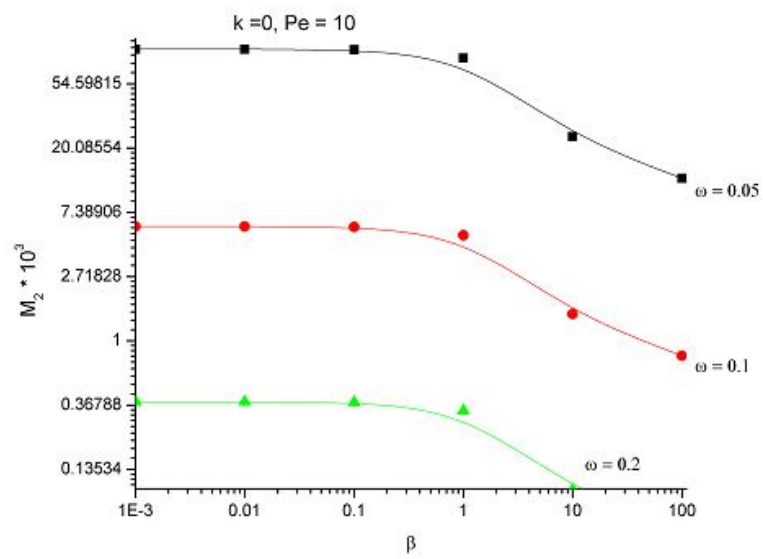

Figure 7

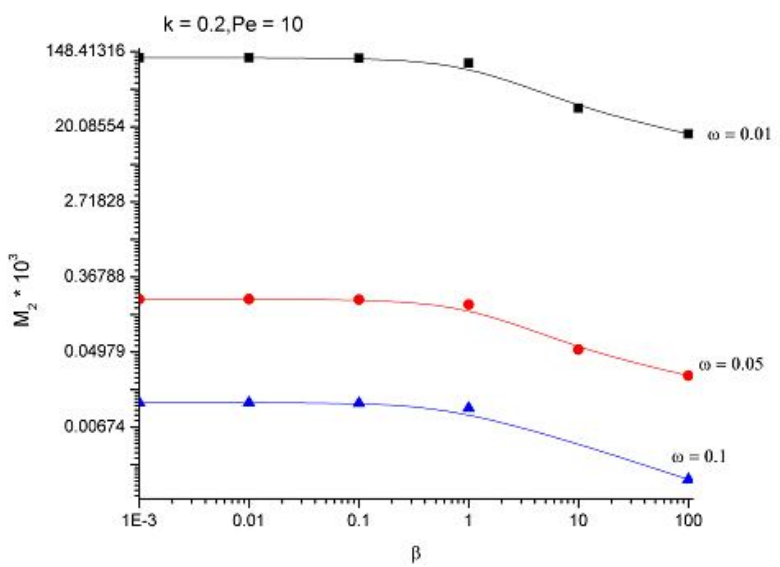

Figure 8

Variation of $M_{2}$ with $\beta$

From figures 7, 8 it can be seen that the asymptotic dispersion coefficient $M_{2}$ decreases with an increase in the wall absorption 
parameter $\beta$ and also drag parameter $\omega$. The effects of wall absorption $\beta$ and annular gap $k$ are less significant comparing to the effect of the drag parameter $\omega$ on the dispersion coefficient $M_{2}$.

\section{Conclusion}

The dispersion of a solute in an concentric annular region is studied using the generalized dispersion model parameter $\beta$. It is found that as absorption parameter increases exchange and convection coefficients will be enhanced, but diffusion coefficient will be reduced. After certain period of time exchange coefficient will be constant for different values annular gap. As the drag parameter increases convection and diffusion coefficients will be reduced. With the enhancement of catheter radius i.e., the annular gap will be reduced then the convection and diffusion coefficients will be decreased.

\section{Acknowledgement}

The encouragement and support from the managements of JSS Academy of Technical Education, Research center M S Ramaiah Institute of Technology , Nitte Meenakshi Institute of Technology and also VTU Grant scheme from Visveswaraya Technical University, Belgaum acknowledged by the authors.

\section{Appendix}

$$
\begin{gathered}
A=\left[\frac{J_{0}(i \omega k)-J_{0}(i \omega)}{Y_{0}(i \omega k) J_{0}(i \omega)-Y_{0}(i \omega) J_{0}(i \omega k)}\right] \\
B=\left[\frac{Y_{0}(i \omega k)-Y_{0}(i \omega)}{J_{0}(i \omega k) Y_{0}(i \omega)-J_{0}(i \omega) Y_{0}(i \omega k)}\right] \\
\mathrm{f}_{0}(\mathrm{r})=\frac{\left(1-\mathrm{k}^{2}\right)}{2} \frac{\mu_{0}\left(\mathrm{Y}_{0}\left(\mu_{0} \mathrm{r}\right) \mathrm{J}_{1}\left(\mu_{0} \mathrm{k}\right)-\mathrm{Y}_{1}\left(\mu_{0} \mathrm{k}\right) \mathrm{J}_{0}\left(\mu_{0} \mathrm{r}\right)\right)}{\left(\mathrm{Y}_{1}\left(\mu_{0}\right) \mathrm{J}_{1}\left(\mu_{0} \mathrm{k}\right)-\mathrm{Y}_{1}\left(\mu_{0} \mathrm{k}\right) \mathrm{J}_{1}\left(\mu_{0}\right)\right)} \\
f_{1}(r)=\sum_{n=0}^{\infty} \frac{A_{1 n} E_{n}\left(\mu_{n} r\right)}{J_{1}\left(\mu_{n} k\right)}
\end{gathered}
$$


Mapana J Sci, 10, 1(2011) The Effect of Particle Drag and Wall Absorption

$$
\begin{gathered}
E_{n}\left(\mu_{n} r\right)=Y_{0}\left(\mu_{n} r\right) J_{1}\left(\mu_{n} k\right)-Y_{1}\left(\mu_{n} k\right) J_{0}\left(\mu_{n} r\right), \\
A_{1 n}=\frac{\int_{k}^{1} r\left(w(r)+M_{0}\right) f_{0}(r) E_{n}\left(\mu_{n} r\right) d r}{J_{1}\left(\mu_{n} k\right)\left(\mu_{n}{ }^{2}-\mu_{0}^{2}\right)}
\end{gathered}
$$

\section{References}

[1] Back, L,H., Kwack, E.Y., Back, M.R., 1996., Flow rate-pressure drop relation in coronary angioplasty: Catheter obstruction effect. Journal of Biomechanical Engineering Transactions, ASME 118, 8389.

[2] Bloomingburg, G. F., Carta, G., 1994., Seperation of protein mixtures by continuous annular chromatography with step elution. The Chem Engn J.55, B19-B27.

[3] Carta, G., Decarli, J. P., Byers, C. H., Sission, W. G., 1989.,Seperation of metals by continuous annular chromatography with step elution. Chem.Engng.Comm. 79,207-227.

[4] Dash, R.K., Jayaraman, G., Mehta, K.N., 1996., Estimation of increased flow resistance in a narrow catheterized artery - a theoretical model. Journal of Biomechanics 29. 917-930.

[5] DeGance, A.E., Johns, L.E, 1980., On the construction of dispersion approximations to the solution of the convective diffusion equation.AIChE.J 26, 411 - 419.

[6] Sankarasubramanian, R., Gill, W.N., !973., Unsteady convective diffusion with interphase mass transfer.Proc. Roy. Soc. Lond. A. 333, $115-132$.

[7] A. Sarkar, G. Jayaraman., 1998., Correction to Flow Rate Pressure Drop Relation in Coronary Angioplasty: Steady Streaming Effect. J. Biomechanics 31,781 - 791 .

[8] A. Sarkar, G. Jayaraman. 2001., Nonlinear analysis of oscillatory flow in the annulus of an elastic tube - application to catheterized artery. Phys. Fluids 13, 2901 - 2912.

[9] Tsui, Y. T., Tremblay, B., 1984., On transient natural convection heat transfer in the annulus between concentric, horizontal cylinders with isothermal surfaces. Int. J. Heat Mass Transfer 27, $103-111$. 